\title{
THE DYNAMICS OF AXISYMMETRIC SLENDER LIQUID BRIDGES BETWEEN UNEQUAL DISKS
}

\author{
José MESEGUER \\ Laboratorio de Aerodinámica, ETSI Aeronáuticos, Universidad Politécnica, 28040 Madrid, Spain
}

Received 22 September 1984; manuscript received in final form 9 October 1985

\begin{abstract}
In this paper the influence of an axial microgravity on the dynamic stability of axisymmetric slender liquid bridges between unequal disks is numerically studied by using a one-dimensional theory. The breaking of such liquid configurations is analyzed and the dependence of some overall characteristics of the breaking process on the value of axial microgravity, the geometry and the volume of the liquid bridge, as well as stability limits are obtained.
\end{abstract}

\section{Introduction}

Small liquid volumes, partially attached to solid boundaries, naturally appear in a large variety of applications. A typical example could be the floating-zone melting method, which has been widely adopted as a crystal growth process for high purity single crystal since the early 1950's.

The size of the melting zone is limited by the gravitational force which overwhelms the surfacetension force as the zone length becomes larger. The development of space laboratories provides a new environment for material processing, and the possibility of growing crystals by the floating-zone method in space has been explored in recent years (a phosphorus-doped silicon crystal has been grown by the floating zone method on board Spacelab-1 [1]).

It is generally accepted that in order to analyze such a complex configuration as the floating zone under microgravity conditions, the first step is to consider its several aspects independently. Thus, floating zone mechanics is studied by assuming that the zone consists of a pure liquid bridge with uniform properties, and held by surface tension forces between two parallel coaxial supports.

Many results concerning the static stability of liquid bridges are well known. Studies of gravityfree situations have been carried out in, for exam- ple, refs. [2-5], and the stability of liquid bridges in a gravitational field is considered in refs. [6-8]. Recently, new attempts have been made to systematize stability analysis of liquid bridges between unequal disks $[9,10]$, which is the configuration that appears in some stages of the available crystal growth methods $[1,11,12]$.

This paper, which can be regarded as a con-

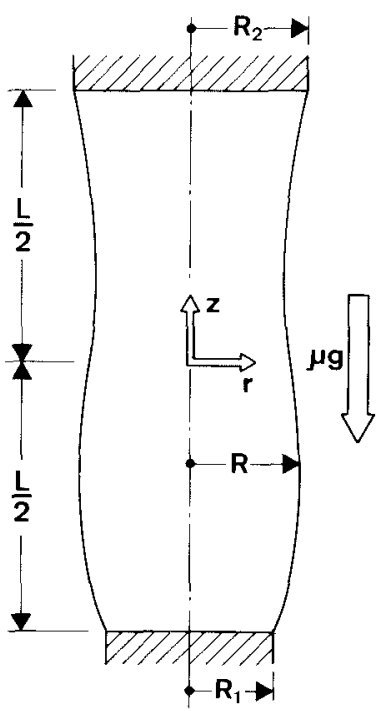

Fig. 1. Geometry and coordinate system for the liquid bridge problem. 
tinuation of a previous work of Meseguer and Sanz [13], is devoted to the hydrodynamics of liquid bridges between unequal disks. The problem to be solved, as sketched in fig. 1, concerns the evolution of an inviscid, axisymmetric, slender liquid bridge, held by surface tension forces between to parallel, coaxial solid disks with different diameters, when disturbed by a microgravitational field acting parallel to the liquid column axis. The problem is analyzed through a non-linear one-dimensional slice model already used in liquid bridge problems.

\section{Mathematical model}

In the following, unless otherwise stated, all lengths are made dimensionless with $R_{0}=\left(R_{1}+\right.$ $\left.R_{2}\right) / 2$, where, as shown in fig. $1, R_{1}$ and $R_{2}$ are the radii of the lower and upper disks, respectively. Volumes are made dimensionless with $R_{0}^{3}$, velocities with $\left(\sigma / \rho R_{0}\right)^{1 / 2}$, time with $\left(\rho R_{0}^{3} / \sigma\right)^{1 / 2}$ and reduced pressure with $\sigma /\left(\rho R_{0}\right), \rho$ being the liquid density and $\sigma$ the surface tension.

The one-dimensional inviscid slice model is generated by assuming that the axial velocity $W$ depends on the axial coordinate $z$ and the time $t$ but not on the radial coordinate $r$. This one-dimensional model has already been used in refs. [13-16] in the analysis of liquid bridge dynamics, and details on numerical integration of the set of differential equations can be found in refs. $[14,15]$. In the case of axisymmetric slender liquid bridges between unequal disks, the problem formulation becomes:

Continuity equation

$S_{t}+Q_{z}=0$.

Axial moments equation

$Q_{t}+\left(Q^{2} / S\right)_{z}=-S P_{z}$,

where $P$, which accounts for both capillary and hydrostatic pressures, is given by

$P=4\left[2 S+\left(S_{z}\right)^{2}-S S_{z z}\right]\left[4 S+\left(S_{z}\right)^{2}\right]^{-3 / 2}+B z$.

In these expressions $S=R^{2}$ represents the cross sectional area (where $R(z, t)$ stands for the equation of the interface shape) and $Q=W S$ the axial momentum of each slice. $B$ is the static Bond number, $B=\rho g R_{0}^{2} / \sigma$, and $g$ the microgravity acceleration. In the following the Bond number is assumed to be positive when microgravity has the sense indicated in fig. 1.

To write down the boundary conditions, two additional nondimensional parameters are introduced, the slenderness of the liquid bridge $\Lambda=$ $L /\left(2 R_{0}\right), L$ being the length of the liquid column, and the lower to upper disk radius ratio $K=$ $R_{1} / R_{2}$. The boundary conditions are

$S( \pm \Lambda, t)=(1 \pm \delta)^{2}, \quad Q( \pm \Lambda, t)=0$,

where $\delta=(1-K) /(1+K)$, and initial conditions

$S(z, 0)=S_{0}(z), \quad Q(z, 0)=0$,

$S_{0}(z)$ being the initial interface shape, which is related to the volume enclosed trough

$V=\pi \int_{-\Lambda}^{A} S_{0}(z) \mathrm{d} z$.

\section{Stability limits}

According to the analytical results obtained in refs. $[10,16]$, the maximum stable slenderness of liquid bridges between unequal disks, having slendernesses of the order of $\pi$ and enclosing a volume close to "cylindrical" volume ( $V=2 \pi \Lambda)$, is given by the expression

$$
\begin{aligned}
\Lambda= & \pi\left[1-(3 / 2)^{4 / 3}\left(B-B_{\mathrm{c}}\right)^{2 / 3}\right] \\
& +\frac{\pi}{2}\left(\frac{V}{2 \pi \Lambda}-1\right),
\end{aligned}
$$

where $B_{\mathrm{c}}=\delta / \pi$. Therefore, if $\Lambda$ and $K$ (or $\delta$ ) are kept constant, the dependence on Bond number of the liquid bridge volume is obtained, as shown in fig. 2. Assuming that liquid bridges with slenderness less than $\pi$ and volumes far from the cylindrical behave in a similar way, minimum volume stability limits can be numerically calculated as follows: each point in the stable region of the $(B, V)$ plane represents a liquid bridge whose interface is in equilibrium under the action of both 


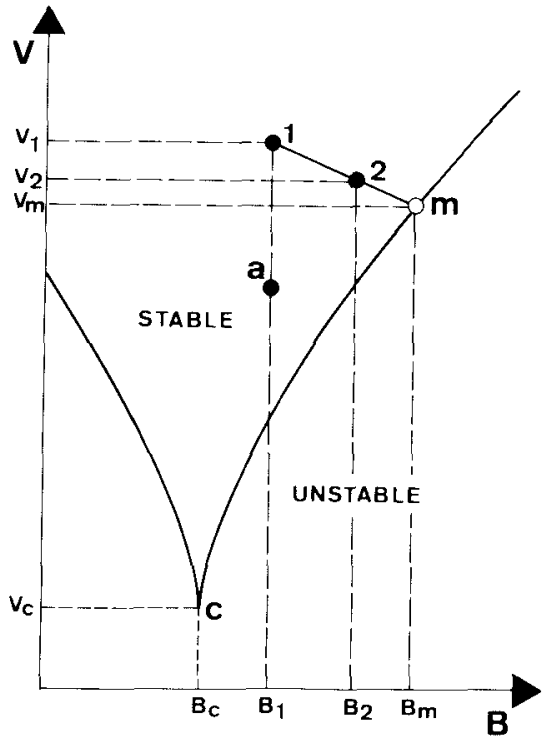

Fig. 2. Stability diagram: minimum volume stability limit $V$ versus Bond number $B$ for liquid bridges between unequal disks.

capillary and hydrostatic pressures. Assume that the liquid bridge configuration represented by point a in fig. 2, which is stable, is perturbed by increasing the Bond number from the initial equilibrium value $B_{\mathrm{i}}=B_{1}$ to a new value $B_{\mathrm{m}}>B_{1}$; if the perturbation $\left(B_{\mathrm{m}}-B_{1}\right)$ is large enough, the liquid bridge will break in two drops whose volume, as well as the time spent by the liquid bridge to reduce its neck radius from its initial value to zero (the breaking time, $t_{\mathrm{b}}$ ) may be calculated by using the one-dimensional slice model.

Once $B_{\mathrm{m}}$ and $B_{1}$ are fixed, the breaking time increases as the liquid volume grows, and there is a value of it, $V_{1}$, for which $t_{\mathrm{b}}$ becomes infinite (see fig. 3). The evolution of a liquid bridge of volume $V$ will be of breaking if $V<V_{1}$ whereas oscillation will occur if $V>V_{1}$. In consequence, $V_{1}$ is just the minimum volume stability limit for the imposed initial condition $B_{1}$ and perturbation $B_{\mathrm{m}}-B_{1}$. To shorten the explanation let us denote this limit as $V_{\mathrm{i}}\left(\Lambda, K, B_{\mathrm{i}}, B_{\mathrm{m}}\right)$.

According to the precedent reasoning, the static minimum volume stability limit would be obtained by considering values of $B_{\mathrm{i}}$ as close as possible to $B_{\mathrm{m}}$, formally when $B_{\mathrm{i}}=B_{\mathrm{m}}$, which is not numerically possible; however, if the difference $B_{\mathrm{m}}-B_{\mathrm{i}}$
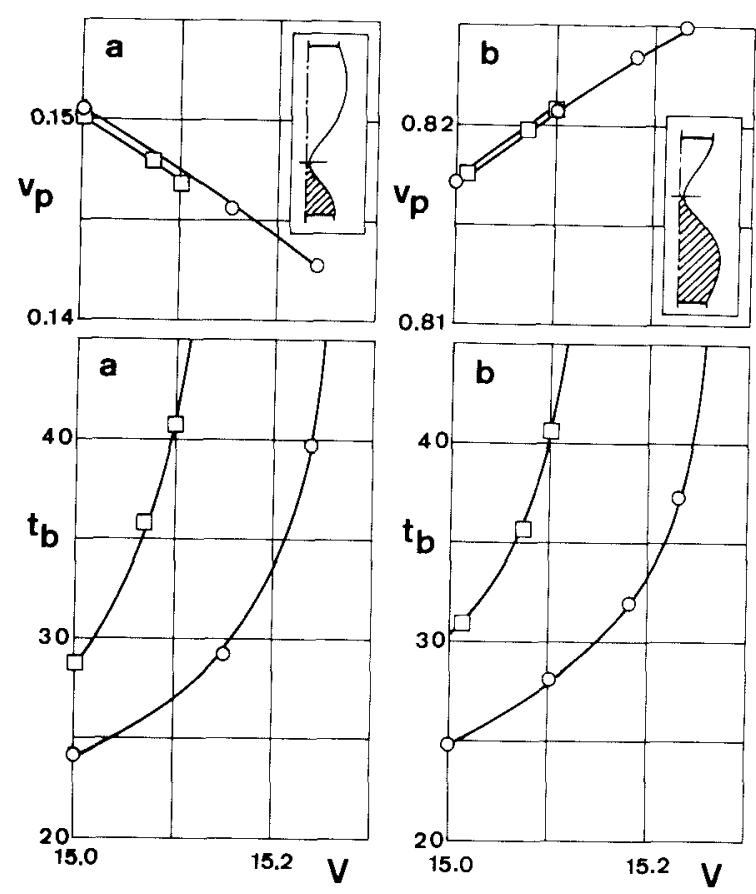

Fig. 3. Variation with the whole volume of the liquid bridge $V$ of the breaking time $t_{b}$ and the partial volume $v_{p}$ of liquid bridges, with a slenderness $A=2.7$, between unequal disks with $K=0.9$. The results in (a) correspond to evolutions of liquid bridges initially in equilibrium under a Bond number $B_{\mathrm{i}}=0.011(\square), 0.014(0)$ which are subjected to a new Bond number $B_{\mathrm{m}}=0.010$. The values for (b) being $B_{\mathrm{i}}=0.039$ ( $\square$ ), $0.036(O)$ and $B_{\mathrm{m}}=0.040$.

is small enough, since $V_{i}\left(A, K, B_{i}, B_{\mathrm{m}}\right)$ varies almost linearly with $B_{i}$, it will be enough to calculate the minimum volume stability limit for two different values of $B_{1}$, let say $B_{1}$ and $B_{2}$ in the plot of fig. 2 , and to extrapolate from these values the static stability limit.

Obviously, the process becomes similar when the static stability limit to be calculated correspond to a Bond number smaller than $B_{c}$, although in this case stable configurations are on the right hand side of the stability limit curve.

\section{Numerical analysis}

Since the problem under consideration depends on four dimensionless parameters: $A, K, V$ and $B$, an analysis of all possible combinations would 
require an enormous computing effort. In consequence, we restricted this numerical analysis to some typical cases, and the dynamics of liquid bridges between unequal disks has been studied for two values of $K(0.9$ and 0.8$)$ and three values of $\Lambda$ (3.0, 2.7 and 2.4).

Numerical results for two representative cases are shown in fig. 3. This plot shows, for several

Table 1

Numerical results: stability limit $V_{\mathrm{i}}$ and partial volume at stability limit $v_{\mathrm{p}}$ of liquid bridges with slenderness $\Lambda$ held between unequal disks with a disk radius ratio $K$; these stability limits have been calculated assuming that liquid bridges are initially at rest under a Bond number $B_{;}$which at the initial time is suddenly changed to the new value $B_{\mathrm{m}}$

\begin{tabular}{|c|c|c|c|c|c|c|c|}
\hline \multicolumn{8}{|l|}{$\Lambda=3.0$} \\
\hline \multicolumn{4}{|l|}{$K=0.9$} & \multicolumn{4}{|c|}{$K=0.8$} \\
\hline$B_{\mathrm{m}}$ & $B_{\mathrm{i}}$ & $Y_{i}$ & $v_{\mathrm{p}}$ & $\overline{B_{\mathrm{m}}}$ & $B_{\mathrm{i}}$ & $V_{\mathrm{i}}$ & $v_{p}$ \\
\hline \multirow[t]{2}{*}{0.010} & 0.014 & 20.14 & 0.105 & 0.030 & 0.034 & 20.39 & 0.089 \\
\hline & 0.011 & 19.91 & 0.109 & & 0.031 & 20.12 & 0.092 \\
\hline \multirow[t]{2}{*}{0.016} & 0.020 & 18.93 & 0.123 & 0.036 & 0.040 & 19.20 & 0.104 \\
\hline & 0.017 & 18.57 & 0.131 & & 0.037 & 18.86 & 0.110 \\
\hline \multirow[t]{2}{*}{0.020} & 0.016 & 18.57 & 0.839 & 0.042 & 0.038 & 19.09 & 0.832 \\
\hline & 0.019 & 18.06 & 0.826 & & 0.041 & 18.66 & 0.822 \\
\hline \multirow[t]{2}{*}{0.030} & 0.026 & 20.87 & 0.878 & 0.050 & 0.046 & 21.10 & 0.866 \\
\hline & 0.029 & 20.38 & 0.873 & & 0.049 & 20.77 & 0.861 \\
\hline
\end{tabular}

\begin{tabular}{|c|c|c|c|c|c|c|c|}
\hline$\Lambda=2.7$ & & & & & & & \\
\hline$K=0.9$ & & & & $K=0$ & & & \\
\hline$B_{\mathrm{m}}$ & $B_{\mathrm{i}}$ & $V_{\mathrm{i}}$ & $v_{\mathrm{p}}$ & $B_{\mathrm{m}}$ & $B_{i}$ & $V_{\mathrm{i}}$ & $v_{\mathrm{p}}$ \\
\hline-0.010 & 0.000 & 17.41 & 0.103 & 0.020 & 0.024 & 17.03 & 0.095 \\
\hline & -0.006 & 17.19 & 0.106 & & 0.021 & 16.90 & 0.097 \\
\hline & -0.008 & 17.11 & 0.107 & & & & \\
\hline 0.000 & 0.004 & 16.22 & 0.122 & 0.040 & 0.044 & 15.14 & 0.129 \\
\hline & 0.001 & 16.11 & 0.124 & & 0.041 & 15.01 & 0.133 \\
\hline 0.010 & 0.014 & 15.26 & 0.142 & 0.050 & 0.054 & 14.12 & 0.160 \\
\hline & 0.011 & 15.12 & 0.147 & & 0.051 & 13.93 & 0.168 \\
\hline 0.020 & 0.024 & 14.21 & 0.175 & 0.056 & 0.052 & 13.94 & 0.755 \\
\hline & 0.021 & 14.06 & 0.181 & & 0.055 & 13.69 & 0.741 \\
\hline 0.024 & 0.026 & 13.61 & 0.201 & 0.060 & 0.056 & 14.48 & 0.779 \\
\hline & 0.025 & 13.52 & 0.206 & & 0.059 & 14.33 & 0.773 \\
\hline 0.027 & 0.024 & 13.64 & 0.762 & 0.070 & 0.066 & 15.69 & 0.819 \\
\hline & 0.026 & 13.46 & 0.752 & & 0.069 & 15.33 & 0.814 \\
\hline 0.030 & 0.026 & 14.09 & 0.785 & & & & \\
\hline & 0.029 & 13.88 & 0.774 & & & & \\
\hline 0.040 & 0.036 & 15.27 & 0.826 & & & & \\
\hline & 0.039 & 15.13 & 0.822 & & & & \\
\hline 0.050 & 0.046 & 16.32 & 0.852 & & & & \\
\hline & 0.049 & 16.19 & 0.849 & & & & \\
\hline 0.060 & 0.056 & 17.39 & 0.871 & & & & \\
\hline & 0.059 & 17.29 & 0.869 & & & & \\
\hline
\end{tabular}




\begin{tabular}{|c|c|c|c|c|c|c|c|}
\hline$A=2.4$ & & & & & & & \\
\hline$K=0.9$ & & & & $K=0$ & & & \\
\hline$B_{\mathrm{m}}$ & $B_{i}$ & $V_{\mathrm{i}}$ & $v_{\mathbf{p}}$ & $\overline{B_{\mathrm{m}}}$ & $B_{\mathrm{j}}$ & $V_{i}$ & $v_{p}$ \\
\hline-0.010 & 0.000 & 12.42 & 0.151 & 0.030 & 0.034 & 12.32 & 0.139 \\
\hline & -0.006 & 12.32 & 0.155 & & 0.031 & 12.27 & 0.140 \\
\hline & -0.008 & 12.29 & 0.156 & & & & \\
\hline 0.000 & 0.004 & 11.86 & 0.172 & 0.060 & 0.064 & 10.98 & 0.198 \\
\hline & 0.001 & 11.79 & 0.175 & & 0.061 & 10.91 & 0.204 \\
\hline 0.020 & 0.024 & 10.94 & 0.221 & 0.070 & 0.074 & 10.50 & 0.237 \\
\hline & 0.021 & 10.87 & 0.226 & & 0.071 & 10.42 & 0.245 \\
\hline 0.030 & 0.034 & 10.47 & 0.260 & & & & \\
\hline & 0.031 & 10.39 & 0.269 & & & & \\
\hline 0.034 & 0.037 & 10.22 & 0.290 & & & & \\
\hline & 0.035 & 10.16 & 0.299 & & & & \\
\hline 0.040 & 0.036 & 10.40 & 0.690 & & & & \\
\hline & 0.039 & 10.32 & 0.679 & & & & \\
\hline 0.050 & 0.046 & 10.93 & 0.741 & & & & \\
\hline & 0.049 & 10.85 & 0.734 & & & & \\
\hline 0.060 & 0.056 & 11.42 & 0.772 & & & & \\
\hline & 0.059 & 11.35 & 0.768 & & & & \\
\hline
\end{tabular}

initial conditions, the variation with the whole liquid bridge volume of both the breaking time $t_{\mathrm{b}}$ and partial volume $v_{\mathrm{p}}$ (defined as the ratio of the volume of liquid enclosed between the bottom disk and the neck of the liquid bridge just before breaking to the whole liquid bridge volume). As already stated, in each of the curves $t_{\mathrm{h}}$ increases as $V$ increases, defining a limiting value $V_{\mathrm{i}}\left(\Lambda, K, B_{\mathrm{i}}\right.$, $B$ ). On the other hand, $v_{\mathrm{p}}$ decreases with $V$ if the Bond number is smaller than $B_{\mathrm{c}}$ or increases if $B>B_{c}$; in both cases $v_{\mathrm{p}}$ results almost independent of initial conditions (provided that breaking perturbations are small enough), depending mainly on the liquid bridge volume.

The results for $\Lambda=3.0,2.7$ and 2.4 are shown in table 1 . In each case the values of $V_{\mathrm{i}}\left(\Lambda, K, B_{\mathrm{i}}\right.$, $B$ ) and $v_{\mathrm{p}}$ have been obtained by plotting the variation of both $t_{\mathrm{b}}$ and $v_{\mathrm{p}}$ versus $V$ as in fig. 3; however these plots are not shown here because they do not represent any additional information. Once $V_{i}\left(A, K, B_{i}, B\right)$ has been obtained for at least two values of $B_{i}$, the static stability limit $V_{\mathrm{m}}$ is calculated by extrapolation. In addition, since $v_{\mathrm{p}}$ varies also almost linearly with $V$, a similar procedure may be used to calculate the value of the partial volume at the static stability limit; these values are shown in table 2 .

The variation with Bond number of both the static minimum volume stability limit and the partial volume have been represented in figs. 4,5 and 6 from data shown in table 2. Concerning stability limits, $V_{\mathrm{m}}$ varies almost linearly with Bond number except in the vicinity of the cusp $\left(B_{c}, V_{c}\right)$, where the slope of the curve increases. Cusps have been calculated by assuming that, close to these points, stability limits behave as in the analytical case, represented in eq. (7). Therefore, since the difference in disk radii plays the role of a nonsymmetric effect (in respect to the middle plane parallel to the disks) like the Bond number, once $\Lambda$ and $K$ are fixed, the dependence of $V_{\mathrm{m}}$ on Bond number may be expressed as

$$
V_{\mathrm{m}}=V_{\mathrm{c}}+d\left(B-B_{\mathrm{c}}\right)^{n},
$$


Table 2

Variation with Bond number $B$ of minimum volume stability limit $V_{m}$ and partial volume at stability limit $v_{\mathrm{p}}$ of liquid bridges with slenderness $A$ held between unequal disks with a disk radius ratio $K$

\begin{tabular}{|c|c|c|c|c|c|}
\hline \multicolumn{6}{|l|}{$\bar{\Lambda}=3.0$} \\
\hline \multicolumn{3}{|l|}{$K=0.9$} & \multicolumn{3}{|c|}{$K=0.8$} \\
\hline$B$ & $V_{\mathrm{m}}$ & $v_{\mathrm{p}}$ & $\bar{B}$ & $V_{\mathrm{m}}$ & $v_{\mathrm{p}}$ \\
\hline 0.010 & 19.83 & 0.110 & 0.030 & 20.03 & 0.094 \\
\hline 0.016 & 18.45 & 0.133 & 0.036 & 18.75 & 0.112 \\
\hline 0.020 & 17.89 & 0.821 & 0.042 & 18.52 & 0.818 \\
\hline 0.030 & 20.48 & 0.872 & 0.050 & 20.66 & 0.859 \\
\hline \multicolumn{6}{|l|}{$A=2.7$} \\
\hline \multicolumn{3}{|l|}{$K=0.9$} & \multicolumn{3}{|c|}{$K=0.8$} \\
\hline$B$ & $V_{\mathrm{m}}$ & $v_{\mathrm{p}}$ & $B$ & $V_{\mathrm{m}}$ & $v_{\mathrm{p}}$ \\
\hline-0.010 & 17.03 & 0.109 & 0.020 & 16.86 & 0.098 \\
\hline 0.000 & 16.07 & 0.125 & 0.040 & 14.97 & 0.134 \\
\hline 0.010 & 15.07 & 0.148 & 0.050 & 13.87 & 0.171 \\
\hline 0.020 & 14.01 & 0.183 & 0.056 & 13.61 & 0.737 \\
\hline 0.024 & 13.43 & 0.211 & 0.060 & 14.28 & 0.771 \\
\hline 0.027 & 13.37 & 0.746 & 0.070 & 15.48 & 0.812 \\
\hline 0.030 & 13.81 & 0.771 & & & \\
\hline 0.040 & 15.08 & 0.820 & & & \\
\hline 0.050 & 16.15 & 0.848 & & & \\
\hline 0.060 & 17.26 & 0.869 & & & \\
\hline \multicolumn{6}{|l|}{$\Lambda=2.4$} \\
\hline \multicolumn{3}{|l|}{$K=0.9$} & \multicolumn{3}{|c|}{$K=0.8$} \\
\hline$B$ & $V_{\mathrm{m}}$ & $v_{\mathrm{p}}$ & $B$ & $V_{\mathrm{m}}$ & $v_{\mathrm{p}}$ \\
\hline-0.010 & 12.26 & 0.157 & 0.030 & 12.25 & 0.141 \\
\hline 0.000 & 11.77 & 0.176 & 0.060 & 10.89 & 0.205 \\
\hline 0.020 & 10.85 & 0.227 & 0.070 & 10.39 & 0.248 \\
\hline 0.030 & 10.36 & 0.272 & & & \\
\hline 0.034 & 10.13 & 0.303 & & & \\
\hline 0.040 & 10.29 & 0.676 & & & \\
\hline 0.050 & 10.82 & 0.731 & & & \\
\hline 0.060 & 11.33 & 0.767 & & & \\
\hline
\end{tabular}

where, in the analytical case, $d=(3 / 2)^{4 / 3}, n=$ $2 / 3$. The values of $V_{\mathrm{c}}$ and $B_{\mathrm{c}}$ have been calculated by fitting eq. (8) to numerical data by a least square method. In this fitting several possibilities appear depending on either $\mathrm{d}$ and $\mathrm{n}$ are assumed to behave as in the analytical case or they are not: both $d$ and $n$ might both behave in this way, or only one of them (the remainder being calculated during fitting) or neither of them. However, although different fittings are possible, the value of $B_{\mathrm{c}}$ results independent of the method employed, and that for $V_{\mathrm{c}}$ almost independent, variations in the value of $V_{c}$ being smaller than $4 \%$ from one method to another. Cusps are shown in table 3, whereas the variation with the slenderness of $V_{c}$ and $B_{c}$ has been plotted in fig. 7. Concerning $V_{c}$ (which is the absolute minimum volume stability limit) it must be pointed out that this limit is, in dimensionless variable, almost the same no matter the value of $K$, at least in the slenderness range under consideration. Therefore, fig. 7 gives as a function of $\Lambda$ the absolute minimum volume stability limit and, for each value of $K$, the Bond number at which this limit is reached. 

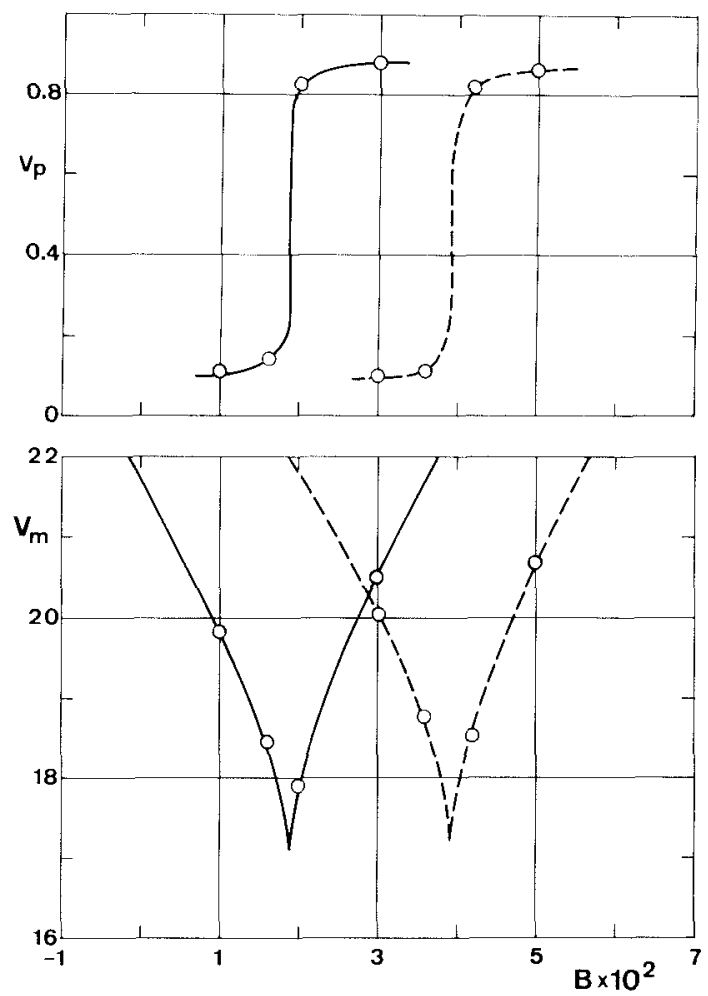

Fig. 4. Static minimum volume stability limit $V_{\mathrm{m}}$ and partial volume at stability limit $v_{\mathrm{p}}$ versus Bond number $B$. The results correspond to liquid bridges with $A=3.0$ held between unequal disks with $K=0.9$ (solid lines) or 0.8 (dashed lines). The circles indicate numerical results.

Concerning partial volumes, as it can be observed in figs. 4, 5 and 6 , when the liquid bridge breaks, the larger drop is obtained at the lower or

Table 3

Absolute minimum volume stability limit $V_{c}$ and Bond number at which this limit is reached $B_{c}$ of liquid bridges with slenderness $\Lambda$ held between unequal disks with a disk radius ratio $K$

\begin{tabular}{llll}
\hline$\Lambda$ & $K$ & $V_{\mathrm{c}}$ & $B_{\mathrm{c}}$ \\
\hline 3.0 & 1.0 & 17.25 & 0 \\
& 0.9 & 17.10 & 0.0187 \\
& 0.8 & 17.20 & 0.0391 \\
2.7 & 1.0 & 13.00 & 0 \\
& 0.9 & 13.00 & 0.0256 \\
& 0.8 & 13.10 & 0.0534 \\
2.4 & 1.0 & 9.92 & 0 \\
& 0.9 & 10.00 & 0.0357 \\
\hline
\end{tabular}
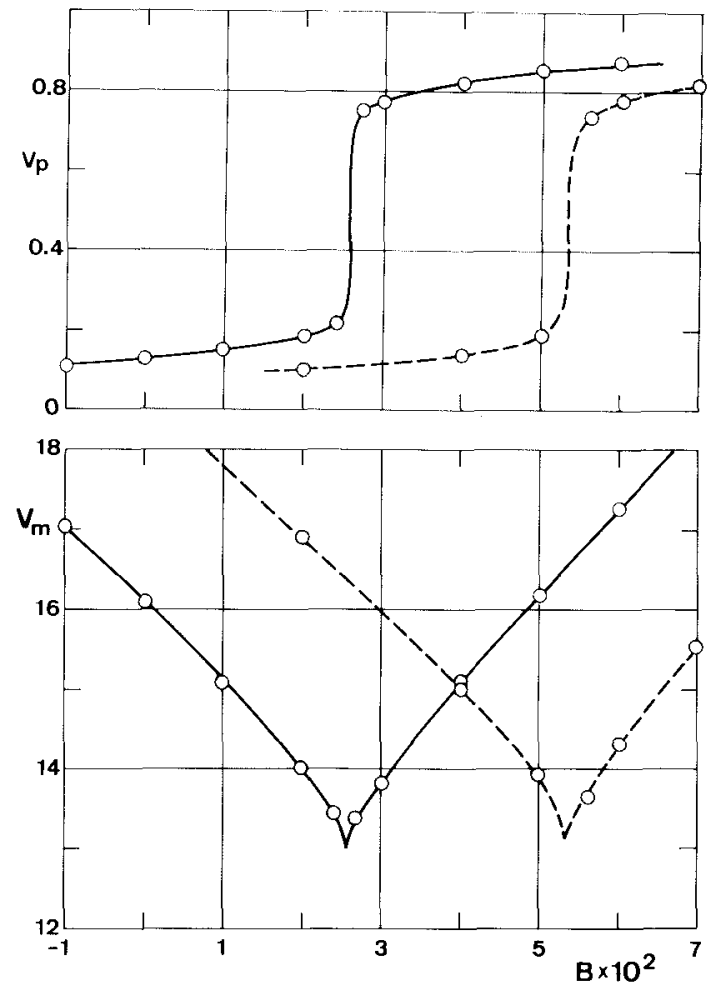

Fig. 5. Static minimum volume stability limit $V_{\mathrm{m}}$ and partial volume at stability limit $v_{\mathrm{p}}$ versus Bond number $B$. The results correspond to liquid bridges with $\Lambda=2.7$ held between unequal disks with $K=0.9$ (solid lines) or 0.8 (dashed lines). The circles indicate numerical results.

the upper disk depending on the value of the Bond number. Within the range of values of $B$ under consideration the breaking of a liquid bridge is mainly driven by surface tension forces, the Bond number playing the role of a perturbation which forces capillary instabilities to develop [15]. Breaking process, thence the volume of the drop resulting from breaking, depends mainly on the initial interface shape (which in turn depends on the initial Bond number $B_{\mathrm{i}}$ ), but not on the breaking perturbation $B-B_{j}$. Numerical results show a dependence on the initial interface shape of the volume of the resulting drops, as it can be observed by comparing the upper plots of figs. 4,5 and 6 with fig. 8 , where the position of the liquid bridge neck has been represented as a function of the initial Bond number for liquid bridges with a slenderness $\Lambda=2.7$ and volumes close to the cor- 

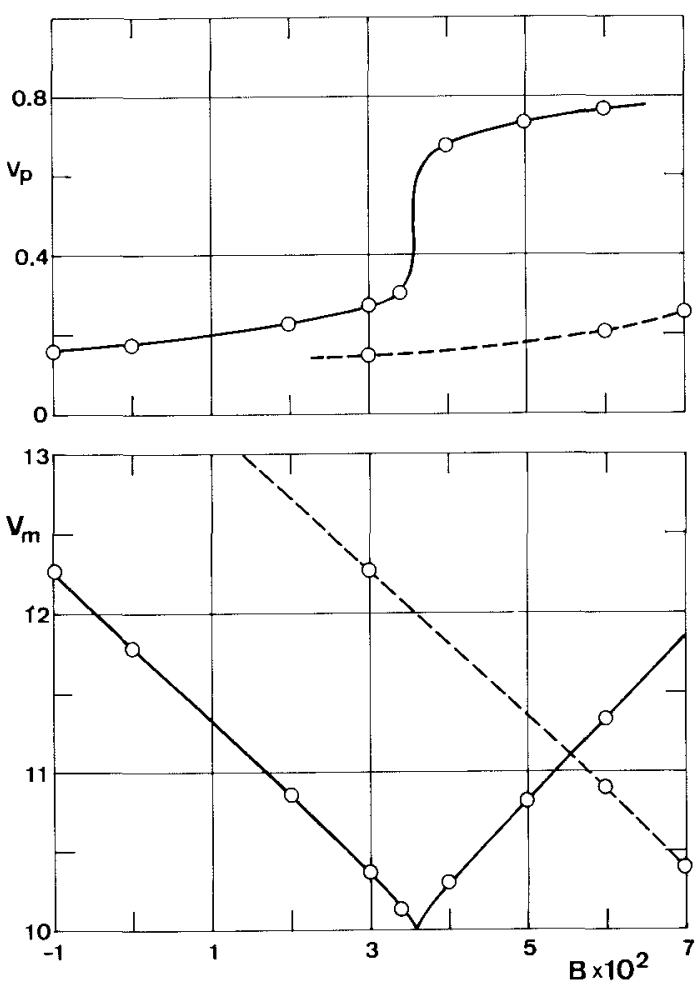

Fig. 6. Static minimum volume stability limit $V_{\mathrm{m}}$ and partial volume at stability limit $v_{\mathrm{p}}$ versus Bond number $B$. The results correspond to liquid bridges with $A=2.4$ held between unequal disks with $K=0.9$ (solid lines) or 0.8 (dashed lines). The circles indicate numerical results.

responding static minimum volume stability limit. The behaviour shown in fig. 8 could be used to experimentally determine the value of the Bond number at the cusp, $B_{\mathrm{c}}$, since close to this point the liquid bridge neck jumps from the bottom half of the liquid bridge to the upper one.

A second aspect to be pointed out from this dynamic analysis is that, in the case of liquid bridges between unequal disks, the Bond number may increase the stability of such configurations, or, in other words, the liquid bridge volume may be smaller than the static minimum volume stability limit corresponding to $B=0$ and the resulting configuration be still stable. This behaviour, which might be surprising, can be explained by thinking that unequal disks is a non-symmetric effect like the Bond number (as stated by eq. (7)). In the case of liquid bridges between equal disks is $B_{\mathrm{c}}=0$ : the

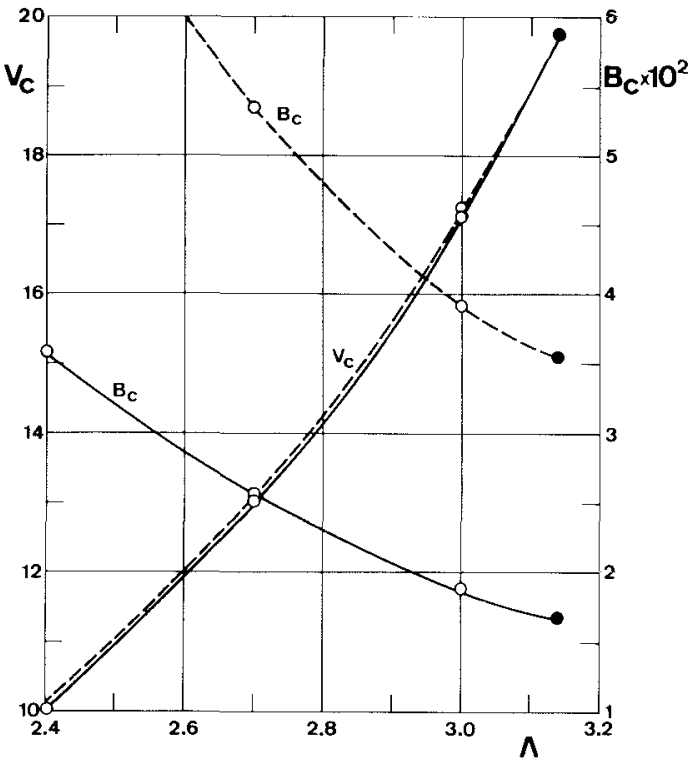

Fig. 7. Variation with the slenderness $\Lambda$ of absolute minimum volume stability limit $V_{c}$ and Bond number at which this stability limit is reached $B_{c}$ of liquid bridges held between unequal disks with $K=0.9$ (solid lines) or 0.8 (dashed lines). White circles correspond to numerical results whereas black ones indicate results obtained from eq. (7).

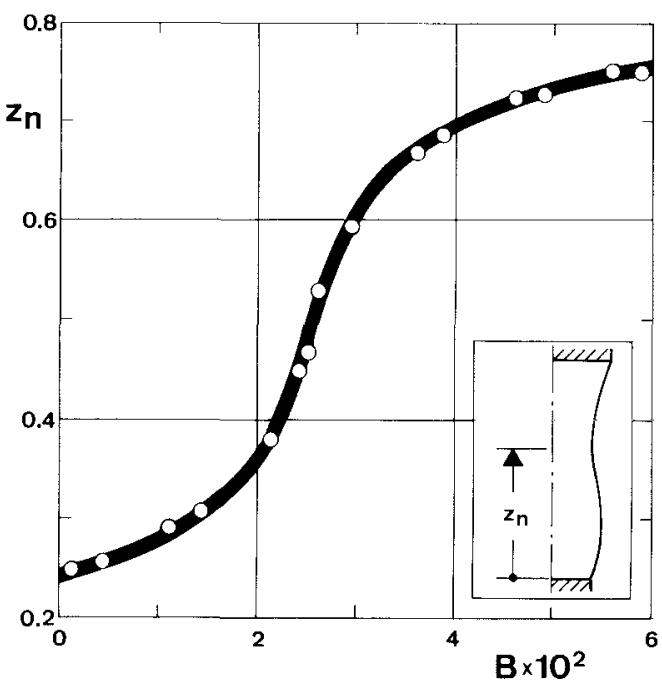

Fig. 8. Variation with Bond number $B$ of the position of the interface neck $z_{n}$ (defined as indicated in the insert) of liquid bridges with $A=2.7$ held between unequal disks with $K=0.9$ and volumes close to the corresponding minimum volume stability limit. The circles indicate numerical results. 
liquid bridge volume must be increased as the Bond number grows to get stable configurations $[8,10,13]$. When $K<1$ the response becomes similar, but now the absolute minimum volume stability limit is reached at $B_{\mathrm{c}} \neq 0$. The static behaviour of liquid bridges between unequal disks is summarized in the stability diagram shown in fig. 9 , which corresponds to liquid bridges with $K=0.9$. A liquid bridge, represented by a point in the $\left(\Lambda, V_{\mathrm{m}}\right)$ diagram, when subjected to a Bond number $B$ is unstable if the point lies under the curve for the specified value of $B$, and stable in the contrary. The different curves have an envelope below which all possible configurations are unstable, this curve being the absolute minimum volume stability limit.

To conclude with this non-linear numerical analysis we can compare, as in fig. 10, numerical results here obtained with eq. (7), which was analytically calculated in ref. [10]; in this plot, the

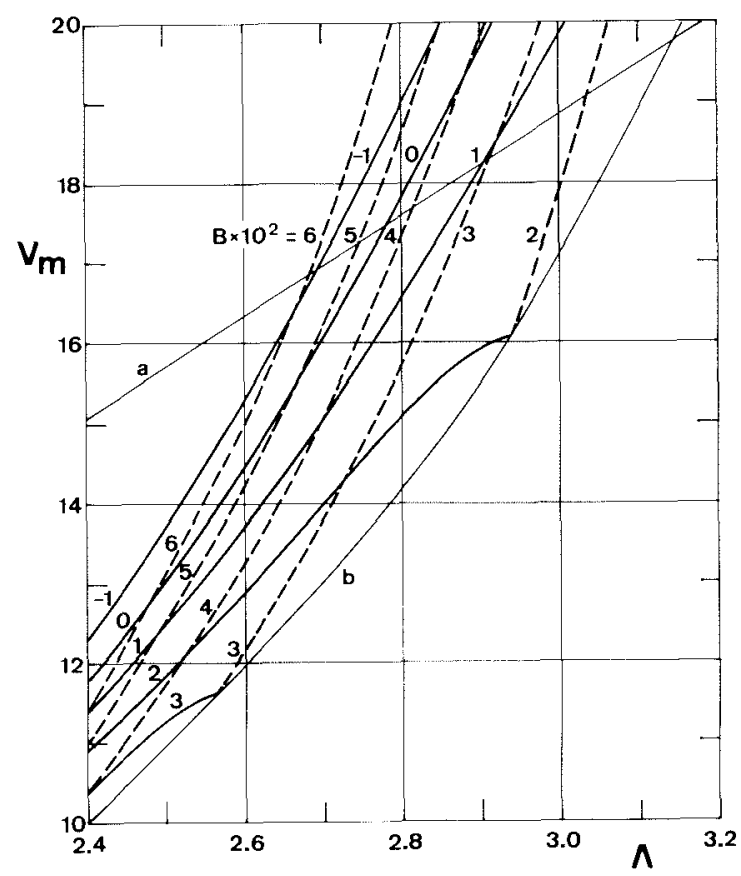

Fig. 9. Stability diagram: minimum volume stability limit $V_{\mathrm{m}}$ versus slenderness $A$ of liquid bridges held between unequal disks with $K=0.9$. Number on the curves indicate the value of the Bond number $B$. The thin solid lines correspond to (a) "cylindrical" liquid bridge configurations, $V=2 \pi \Lambda$, and (b) absolute minimum volume stability limit.

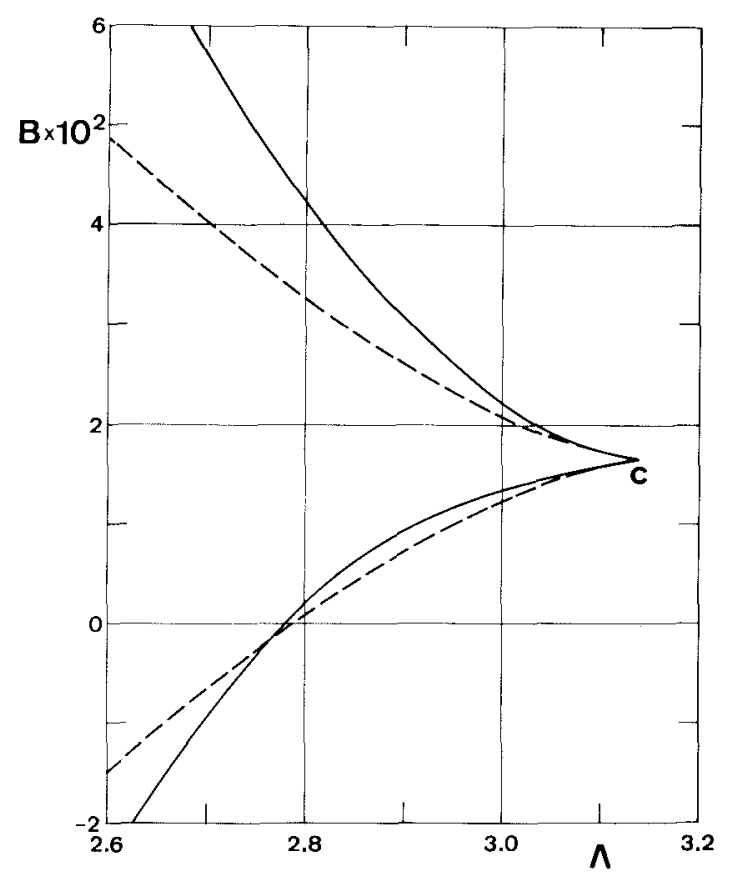

Fig. 10. Maximum stable slenderness $A$ versus Bond number $B$ of liquid bridges between unequal disks $(K=0.9)$ with "cylindrical" volume $(V=2 \pi \Lambda)$. Continuous lines correspond to numerical results whereas dashed ones indicate results obtained îrom eq. (7).

variation with Bond number of the maximum stable slenderness of liquid bridges having cylindrical volume $(V=2 \pi \Lambda)$, slenderness close to $\pi$ and $K=0.9$, has been represented. As it can be observed, if $B>B_{\mathrm{c}}$ numerical results give higher values of the stable slenderness than the analytical case, whereas the contrary occurs when $B<B_{c}$. (provided that $B$ is close enough to $B_{\mathrm{c}}$ ). The explanation of this behaviour may be found in the meaning of the imposed perturbations. It has been said that both $B$ and $K$ are non-symmetric perturbations, therefore the addition of both perturbations can be treated as a single perturbation of the same character, $B_{\mathrm{c}}$ being the origin for this global perturbation. Under such a circumstance, the behaviour predicted by eq. (7) is the same no matter the perturbation is smaller or greater than $B_{c}$. However, according to numerical results, the liquid bridge behaviour is modified by the sense of the resulting perturbation: because of necking effects, stability decreases faster when the resulting perturbation points out to the larger disk. 


\section{Conclusions}

The stability of liquid bridges between unequal disks has been studied under a dynamic point of view by using a non-linear, one-dimensional, inviscid slice model. Stability diagrams have been obtained as well as some other characteristics, either static or dynamic, which are suitable to be experimentally checked in further developments. In this paper only slender liquid bridges have been treated and only two values of $K$ have been considered, although extending the results to other values of $\Lambda$ and/or $K$ (if these are within the range of validity of the slice model) is only a problem of computing effort.

\section{Acknowledgements}

This work has been supported by the Spanish National Commission for Space Research (CONIE) under a contract with the Polytechnic University of Madrid (UPM). I gratefully acknowledge helpful discussions with Dr. A. Sanz and Dr. I. Martínez.

\section{References}

[1] A. Eyer, H. Leiste and R. Nitsche, in: Material Sciences under Microgravity ESA-SP 222 (European Space Agency, Paris, 1984).

[2] J.M. Haynes, J. Colloid Interface Sci. 32 (1970) 652.

[3] J.A. Erle, R.D. Gillette and D.C. Dyson, Chem. Eng. J. 1 (1970) 97.

[4] R.D. Gillette and R.C. Dyson, Chem. Eng. J. 2 (1971) 44.

[5] I. Da Riva, and I. Martinez, in: Material Sciences in Space ESA SP-142 (European Space Agency, Paris, 1979).

[6] S.R. Coriell and M.R. Cordes, J. Crystal Growth 42 (1977) 466.

[7] S.R. Coriell, S.C. Hardy and M.R. Cordes, J. Colloid Interface Sci. 60 (1977) 126.

[8] J.M. Vega and J.M. Perales, in: Material Sciences under Microgravity ESA SP-191 (European Space Agency, Paris, 1983).

[9] I. Martínez, in: Material Sciences under Microgravity ESA SP-191 (European Space Agency, Paris, 1983).

[10] J. Meseguer, J. Crystal Growth 67 (1984) 141.

[11] A. Eyer, B.O. Kolbesen and R. Nitsche, J. Crystal Growth 57 (1980) 145.

[12] H.M. Ettouney, R.A. Brown and J.P. Kalejs, J. Crystal Growth 62 (1983) 230.

[13] J. Meseguer and A. Sanz, J. Fluid Mech. 153 (1985) 83.

[14] J. Meseguer, J. Fluid Mech. 130 (1983) 123.

[15] J. Meseguer, J. Crystal Growth 62 (1983) 577.

[16] D. Rivas and J. Meseguer, J. Fluid Mech. 138 (1984) 417. 